\title{
Face Description with Local Invariant Features: Application to Face Recognition
}

\author{
Sanjay A. Pardeshi, \\ Rajarambapu Institute of Technology, Rajaramnagar \\ Sangli (M.S.), India
}

\author{
Dr. S.N. Talbar, \\ S.G.G.S.C.O.E.T, Nanded \\ Nanded (M.S.), India
}

\begin{abstract}
A completely automatic face recognition system is presented. The method works on color face images and localizes the face region from them initially. It then determines and selects important fiducial facial points and characterizes them by applying a bank of Gabor filters which extract the peculiar texture around them (jets). A well known PCA technique is used to reduce the dimensionality of jets and recognition is realized by measuring the similarity between different jets in eigenspace. The system design is inspired by recent advancements in local feature detection and feature extraction techniques. A complete investigation on the proposed system is conducted, which covers face recognition under pose, illumination and expression variations. The performance of the proposed system is compared with standard methods and it shows the superiority of the proposed system. This research also demonstrates that the face image can be completely characterized with 125 fiducial facial feature points and suggests that L1-norm distance metric is most suitable to measure image similarity in eigenspace. The proposed system reduces the feature vector dimensionality considerably. It results in reduced computational cost and storage cost. In addition to this, proposed system is very robust to all types of image variations. All these benefits make the proposed system most suitable for machine face recognition application.
\end{abstract}

The full text of the article is not available in the cache. Kindly refer the IJCA digital library at www.ijcaonline.org for the complete article. In case, you face problems while downloading the full-text, please send a mail to editor at editor@ijcaonline.org 